\title{
SY15-1 Symposium
}

\section{The KEAP1-NRF2 System for Understanding of Diseases and Drug Development}

\section{Masayuki Yamamoto}

\author{
Department of Medical Biochemistry, Tohoku University Graduate School of Medicine, Japan
}

Our body has an ability to sense quickly environmental insults and to activate cellular defense enzyme genes. Transcription factor NRF2 is essential for the coordinated induction of cellular defense enzymes and protection of tissues. This notion has been supported by experiments using animal models, showing that Nrf2-deficient mice are sensitive to a wide variety of toxic electrophiles and reactive oxygen species (ROS). We found KEAP1 that acts as a subunit of ubiquitin-E3 ligase and degrades NRF2 constitutively. Keap1 also acts as a sensor for electrophilic and oxidative stresses. Covalent modifications of the cysteine residues of KEAP1 abrogate the ubiquitin ligase activity and stabilize NRF2, as if Floodgate opens in response to environmental stresses. This system has been referred to as the Cysteine Code and KEAP1. The two-site recognition / hinge-latch model has also been proposed for the KEAP1-NRF2 system. Disruption of the two-site recognition model explains the mechanism of nuclear accumulation of NRF2 in a Cul3-KEAP1 E3 ubiquitin ligase-dependent manner. We have verified this model through structure biology, mouse genetics, and human disease analyses. Meanwhile, NRF2 inducers are shown to be important for the treatment of stress-based diseases. Especially, NRF2 acts to suppress inflammations through repressing pro-inflammatory cytokine gene expressions and also suppress oxidative tissue damage through inducing a set of antioxidant enzyme genes. Of note, many somatic missense mutations have been identified in KEAP1 and NRF2 genes of human cancers. These mutations disrupt the KEAP1-NRF2 complex and result in constitutive activation of NRF2. Subsequently, elevated expression of NRF2 target genes confers advantages on the growth of cancer cells through the metabolic reprogramming and induction of cellular defense enzymes. Thus, NRF2 inhibitor is now important for the chemo-sensitization therapy of cancers. The KEAP1-NRF2 system opens a new avenue to the understanding of the signal transduction and regulatory processes underlying the stress response and cancer progression. 